\title{
Une réserve littorale pour le Tell septentrional tunisien
}

Pierre FARNOLE

Docteur en océanologie - ERAMM conseil en environnement et génie côtier(Sophia Antipolis)

\section{Résumé}

L'objectif est de définir un plan d'action pour le littoral du Tell Septentrional à court et moyen terme (horizon 2016) et les projets prioritaires. L'étude est réalisée pour le Ministère de l'Economie Tunisien par un groupement de bureaux d'études Tuniso-Belges dont BELGROMA est mandataire commun. Notre intervention concerne uniquement le volet littoral. Le littoral tunisien est soumis à une pression anthropique forte qui nuit à la préservation des écosystèmes côtiers. Il devient donc nécessaire, dans les zones restées vierges et ayant un fort potentiel écologique, d'éviter la création d'infrastructures lourdes qui nuisent à ces équilibres. Le projet d'action pour les 20 prochaines années est axé vers la création d'une réserve littorale et maritime qui inclus tout ou partie du bassin versant littoral et son prolongement dans la zone infralittorale.

\section{Abstract}

Aim is to define an action plan for the coastal zone of the "Tell Septentrional $»$ at shot-range and long-range (to 2016) and the priorities projects. These studies are realised for the economical Tunisian Ministry by a consulting group Tunisian and Belgium wich BELGROMA is the leader. Our expert's reports concern uniquely the coastal zone. This are submitted to a strong anthropic pressure on the coastal ecosystem. It should be necessary to preserved the coastal zone with an important ecological potential. For the twenty next years, the most important project is to create a coastal reserve which included the land basin and the subtidale zone.

\section{Introduction}

Le cadre de l'étude réalisée par le groupement Belgroma, Abdelkafi et Concept ingénierie repose sur l'approche méthodologique suivante:

- une stratégie de développement intégrée ;

- l'obtention d'un consensus des principaux acteurs sur la stratégie à suivre ;

- la rédaction d'un plan d'action à court, moyen et long terme avec indication des projets prioritaires;

- la mise en place d'une approche participative intégrée.

Sur le volet littoral, les objectifs qui ont été définis sont d'assurer une protection efficace de la frange littorale à la fois maritime et continentale contre toute 
tentation d'exploitation anarchique qui ne pourrait avoir qu'un impact négatif sur le patrimoine paysager et écologique original. II s'agit de limiter les implantations urbaines, touristiques et industrielles afin de préserver la biodiversité mais aussi réduire les risques de pollution de la ressource en eau. L'étendue de la zone à protéger correspond au débouché de l'oued Berkoukech en limite occidentale et Ras El Qouran en limite orientale, soit environ $100 \mathrm{~km}$ de littoral (Figure 1). Dans ce cadre, notre mission a été de traiter le volet «littoral» de ce territoire au niveau de la rédaction d'un plan d'action à court, moyen et long terme.

Les orientations qui sont données en matière d'aménagement et de protection sont les suivantes:

- création d'une réserve littorale

- création d'une réserve maritime pour la pêche

- créer un centre de recherche océanographique et de la ressource halieutique

- développer un écotourisme (sentier littoral, chemin de Grandes Randonniées, activités équestres, activités nautiques et sub-aquatiques)

Sur le plan de l'urbanisation et des infrastructures, cette région est peu développée. L'activité agro-sylvo-pastorale y est encore très intense et nuit aux écosystèmes.

\section{Cadre physique et naturel}

Le Tell septentrional de la Tunisie couvre une vaste région qui s'étend de la frontière algérienne à l'Ouest à ras el Qouran à l'Est (Figure 1). Trois facteurs interviennent de façon déterminante sur le climat : le relief, la mer et les vents dominants. Les précipitations atteignent $800 \mathrm{~mm} / \mathrm{an}$ dans cette région qui est considérée comme le "château d'eau » de la Tunisie. Le ruissellement important (taux atteignant $80 \%$ ) sur les formations marno-calcaires et le flysch gréseux abouti à une érosion intense des sols que l'on peut observer sur toute la frange littorale (Photo 1). Le volume annuel ruisselé est de $850 \mathrm{M}^{\mathrm{l}} \mathrm{m} 3$ dont $75 \%$ sont mobilisables pour la ressource en eau (Réf.1). Celle-ci est d'excellente qualité chimique (salinité $<\lg /$ ) et relativement peu chargée en sédiment (concentration moyenne de $5 \mathrm{~g} / \mathrm{l})$. La demande très forte en eau potable pour l'ensemble du pays a conduit les autorités tunisiennes à réaliser d'importants ouvrages hydrauliques dans la région étudiée afin de se constituer des réserves en eau potable suffisantes à 1'horizon 2020. Parmi le plus important d'entre eux, celui en cours de construction sur l'oued El Barrek. A terme, les aménagements hydrauliques doivent permettre la récupération de $85 \%$ des eaux ruissellées.

\footnotetext{
${ }^{1}$ Million
} 


\subsection{Site de Cap Serrat}

Ce site, bien protégé des houles dominantes d'WNW, abrite des petites criques qui peuvent servir de zone de mouillage (Photo 2). Il est remarquable du point de vue paysager avec l'avancée de cap Serrat et sa végétation particulière sur une formation rocheuse très instable (éboulements rocheux) qu'il est nécessaire de stabiliser. La plage de sable fin sur laquelle débouche l'oued est large et le cordon dunaire bordier se prolonge sur les collines littorales (Photo 3). L'écosystème estuarien est riche (végétation, faune, avifaune..) mais risque d'être dégradé avec la construction d'un barrage.

\subsection{Site de Kef Abed}

Kef Abed est un village de pêcheur. Son littoral est composé d'une plage de sable fin (Photo 4). La plage est relativement étroite et le stock sédimentaire réduit en raison de la présence de platiers rocheux en bas-estran. L'origine des sables est en partie locale, issue de la désagrégation des formations gréseuses et des apports solides des oueds. Le cordon dunaire, perché jusqu'à $20 \mathrm{~m}$ à $30 \mathrm{~m}$ d'altitude s'appuie sur le relief en pente douce orienté WNW-ESE qui est la direction principale du vent local. Le site est très exposé aux flux maritimes d'W avec la présence de nombreux hauts fonds dangereux pour la navigation.

\subsection{La dune de NEFZA OUCHTATA}

Les dépôts éoliens se divisent en deux systèmes, les dunes anciennes fixées par la végétation arbustive et la dunes vives migrant vers l'Est. Les dunes littorales couvrent environ 10000 ha répartis sur les 3 Gouvernorats du Nord de la Tunisie. La plus importante se situe dans le Gouvernorat de Béja avec 5200ha dont 4 400 ha entièrement fixée. Les dunes anciennes sont colonisées par : quercus suber, Pistacia lentiscus, Philityrea media, Rhamnus, Asparagus acutifolius, Geranium robertianum ssp purpureum, Calycotome villosa

Les dunes en voie de fixation sont colonisées par: juniperus eccycedirus, Juniperus phoenica, Retama monosperma

La particularité du milieu dunaire est son étendue et son instabilité. Il constitue un espace naturel riche qu'il convient de préserver pour plusieurs raisons:

- la protection naturelle contre l'érosion marine ;

- la stabilisation du trait de côte ;

- la valeur archéologique puisqu'elles recèlent des outillages en silex d'âge néolithique (Réf.4) 
Photo 1 : Erosion des sols sur le flysch gréseux (Mogods)

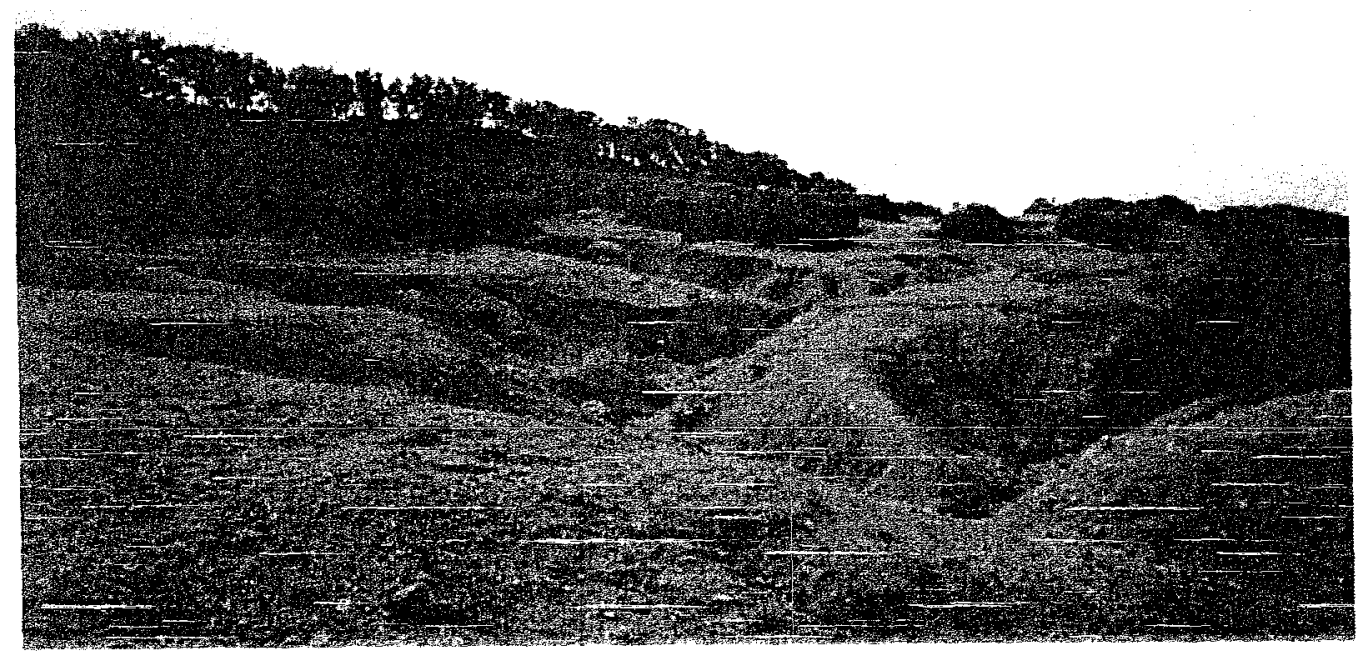

Photo 2 : Cap Serrat

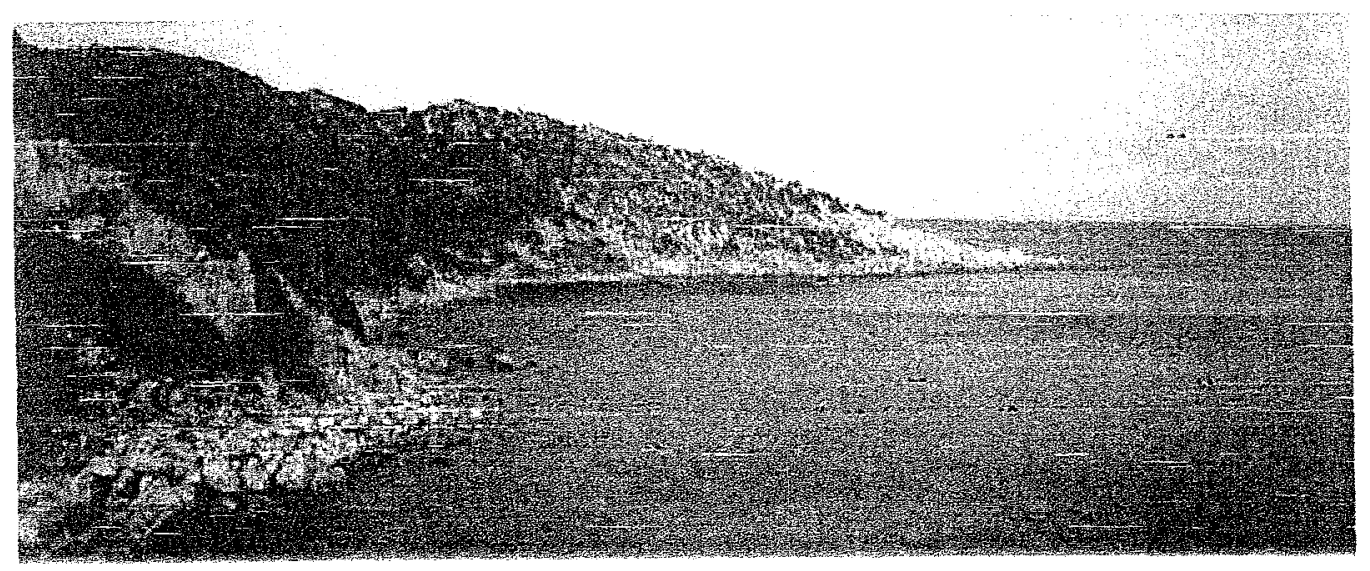

Photo 3 : Plage de Cap Serrat avec en fond de plan les dunes perchées de Kef Abed

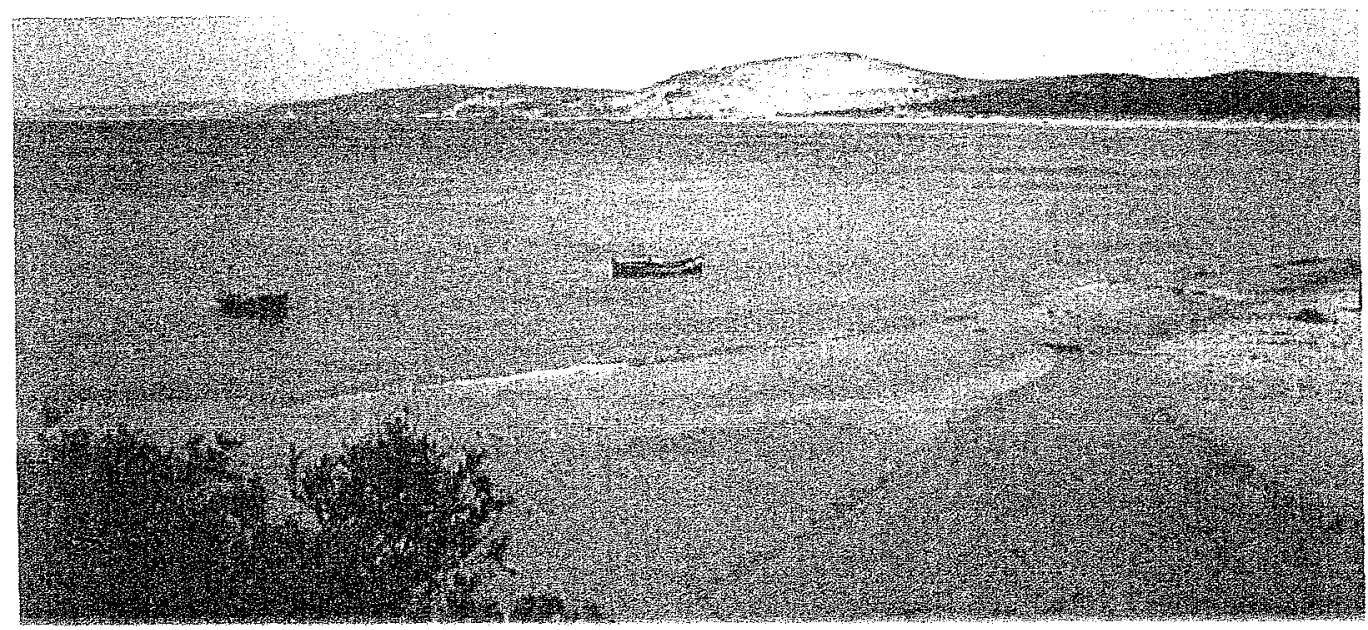


Photo 4 : Plage de Kef Abed

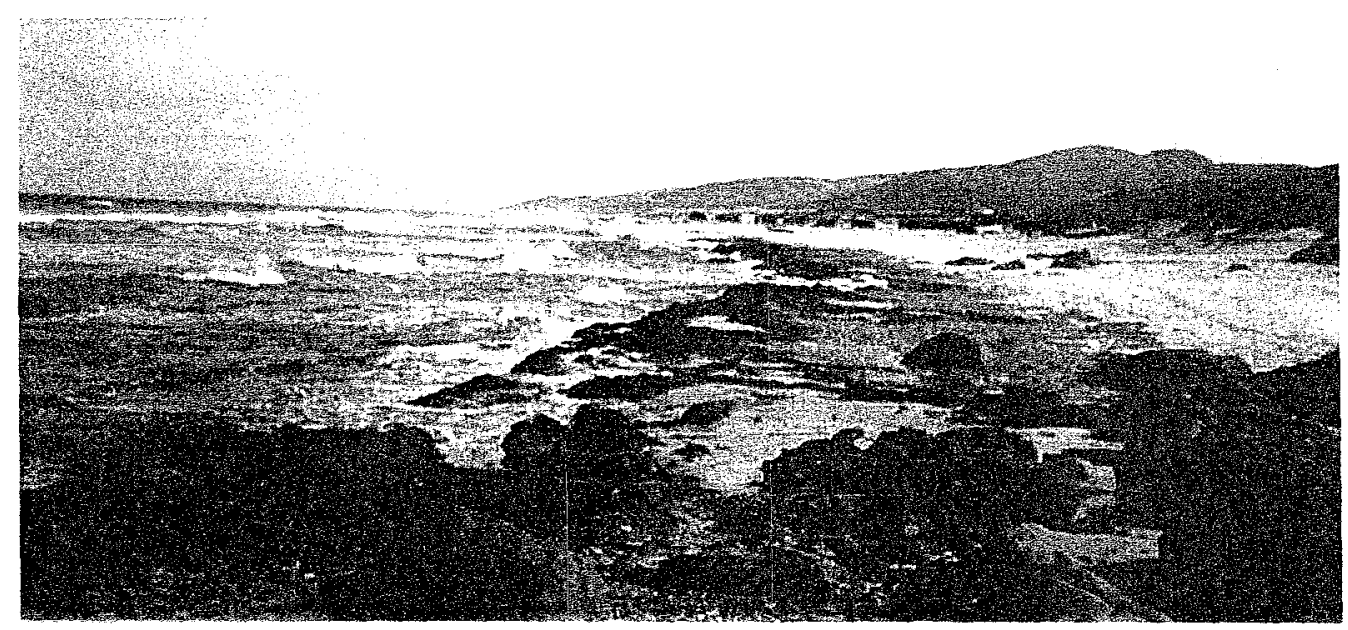

Photo 5 : Barrage en construction sur l'oued Sidi El Barrek (Zouaraa)

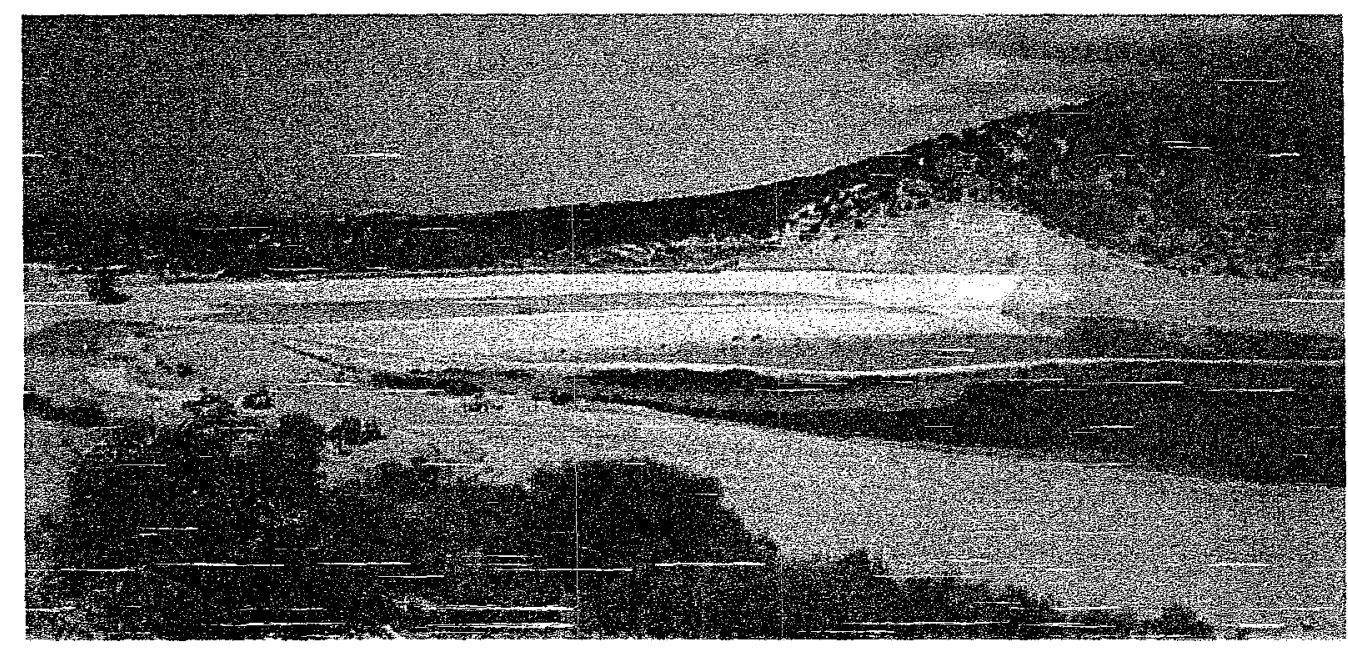

Photo 6: Oued El Barrek envasé avec un bassin versant constitué de dunes vives en voie de fixation

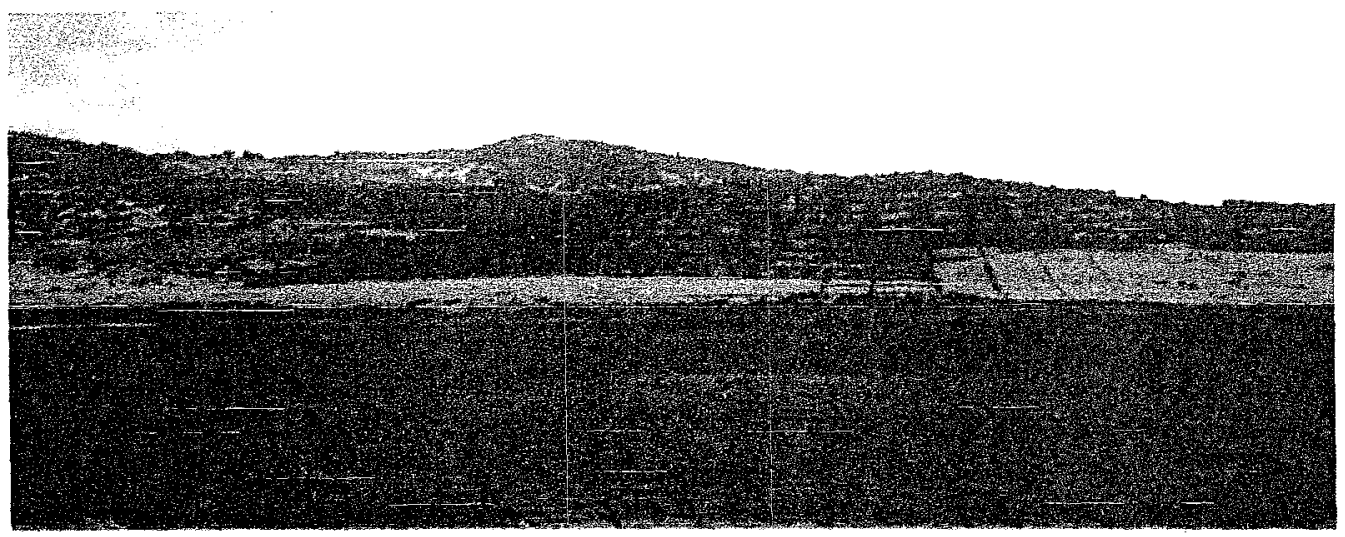




\subsection{La gestion du milieu dunaire}

Le procédé de fixation des dunes vives suit un cheminement inverse de celui de la progression des dunes. La fixation a débuté dès 1953 sur la partie la plus continentale avec un front d'avancement des travaux vers la mer. Dès 1973, la fixation des dunes vives a été entreprise au niveau de la source d'alimentation, c'est-à-dire coté mer. Le procédé consiste à installer une haie de branchage de chêne-kermes et de genévriers en limite de haut de plage selon une ligne parallèle au trait de côte. Au fur et à mesure du recouvrement par le sable, une nouvelle haie vient s'installer sur la première. Le profil d'équilibre est atteint vers $14 \mathrm{~m}$ de hauteur. Plusieurs tranchées sont ainsi réalisées, mais la dune reste vive. Il faut donc la fixer toujours à l'aide de branchages par un système de clayonnage dont les dimensions dépendent de l'exposition au vent et de la pente (variant de $10 \mathrm{~m} \times 10 \mathrm{~m}$ juisqui'à $40 \mathrm{~m} \times 20 \mathrm{~m}$ Photo 6). La troisième étape consiste à planter le cordon dunaire avec deux espèces d'acacias : acacia cyclopis à moins d' $1 \mathrm{~km}$ de la mer et acacias cyanophylla à plus de $1 \mathrm{~km}$. Les résultats obtenus avec cette technique sont remarquables. Le seul handicap est le pacage illicite par les ovins et bovins. L'acacia cyanophylla est exploité entre 8 et 10 ans puis il cède sa piace au pin pignon ou d'autres espèces de pins, le pin maritime ou le pin radiata ce dernier donne un excellent rendement. Dans les points bas, l'eucalyptus gomphocepha et camaldulensis donnent de bon rendement.

\subsection{L'aménagement des barrages}

Les barrages construits sur les oueds ont d'autant plus d'influence sur l'équilibre sédimentaire littoral que leur bassin versant est étendu. Sur un site comme Zouaraa (barrage de Sidi El Barrek), il est à craindre une forte incidence sur cet équilibre. Les études mentionnent des superficies d'eaux stagnantes correspondant à $22,5 \%$ de la totalité de la retenue sur 50 ans (Réf.8). En terme d'envasement, l'estimation des dépôts est de $58 \mathrm{Mm} 3$, soit un envasement moyen annuel de l'ordre d' $1 \mathrm{Mm} 3$. Si l'on considère que la fraction vaseuse représente $50 \%$, on peut s'attendre à un déficit moyen annuel en sédiments pour la zone littorale de l'ordre de $500000 \mathrm{~m} 3$ qu'il sera nécessaire à terme de gérer par un ré engraissement artificiel du littoral à partir des importants stocks disponibles à l'intérieur des terres.

\section{Projet de réserve littorale}

\subsection{Identification des enjeux}

Le littoral tunisien est soumis à une pression touristique forte qui nuit à la préservation des écosystèmes côtiers. Il devient donc nécessaire, dans les zones restées vierges et ayant un fort potentiel écologique de diversifier les activités. C'est ce qui est proposé pour le Tell septentrional. Une réserve littorale ne doit pas être terrestre ou maritime (Figure 1). Elle doit inclure tout ou partie du bassin 
versant littoral et son prolongement dans la zone infralittorale. Cette unité morphologique ne doit pas être scindée, car elle constitue la principale richesse écologique de cet espace. Un déséquilibre du milieu littoral aura des répercussions sur la zone infralittorale et inversement. Il est donc souhaitable dans le cadre de la mise en place d'un réserve littorale dintégrer une zone maritime et continentale qui soit considérée comme homogène.

\subsection{Les limites de la zone protégée}

\section{Limite Ouest:}

Débouché de l'Oued Berkoukech (409 Lat; 406 Lon): L'étendue de la zone protégée est en moyenne de $10 \mathrm{~km}$ selon un axe transversal à la côte incluant $5 \mathrm{~km}$ dans la partie littorale terrestre et entre $5 \mathrm{~km}$ et $10 \mathrm{~km}$ dans la zone infralittorale.

\section{Limite Est:}

Cap Ras El Qouran (445 Lat; 470 Lon). Le linéaire côtier est de 90 km entre ces deux limites.

\begin{tabular}{|c|c|c|}
\hline Réserve littorale & Superficies en $\mathrm{km} 2$ & Echéancier \\
\hline Terrestre & 374 & 2011 \\
\hline Maritime & 772 & 2016 \\
\hline
\end{tabular}

\subsection{Les produits attendus}

La valorisation et la préservation des écosystèmes littoraux ne constituent pas une ressource économique directe. Elles ne cherchent pas à développer une fréquentation excessive d'un site remarquable par ses paysages et son écosystème. Celle-ci n'est pas compatible avec une bonne gestion du milieu naturel. Une trop grande facilité d'accès serait nuisible. Par contre, on peut envisager le développement d'un écotourisme non dégradant pour le milieu naturel et susceptible de constituer une ressource économique.

L'APAL (Agence pour la Protection et l'Aménagement du Littoral) est favorable à ce type d'aménagement pour les zones sensibles. L'aménagement des pistes existantes en réseau routier entre Sidi Mechreg et Cap Serrat n'est pas souhaité. Les accès à la mer doivent être privilégiés selon un axe transversal au rivage. Les accès par voie maritime devraient être développés grâce à la création de mouillages organisés sur des sites abrités en substitution à la construction de nouvelles infrastructures portuaires.

Le littoral du Tell septentrional étant considéré comme zone sensible, il revient à 1 'APAL, organisme gestionnaire des zones sensibles, de mettre en place et de gérer le projet de «Réserve Littorale ». 


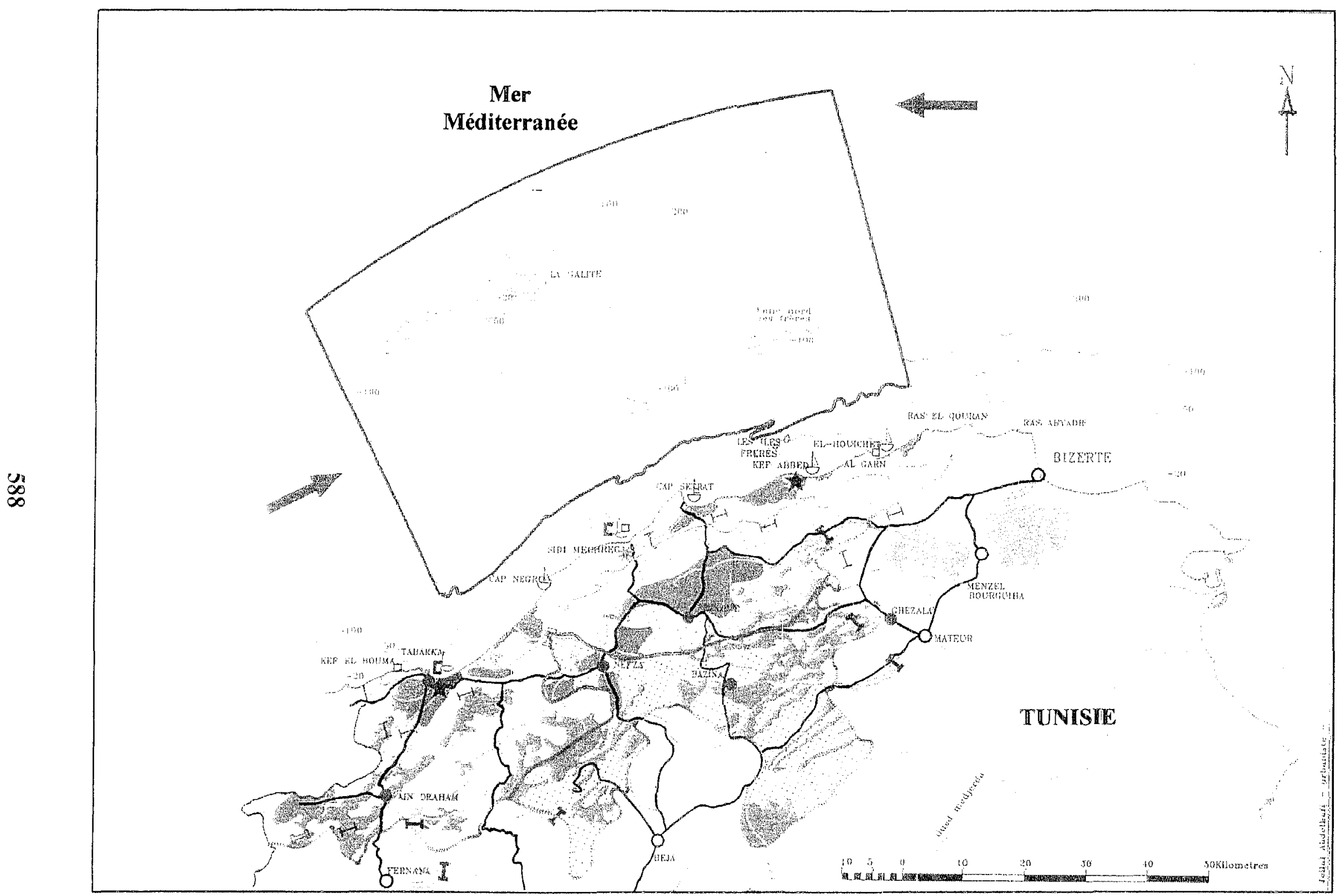

בิ

Figure 1 : Localisation de la zone d'étude et des projets 


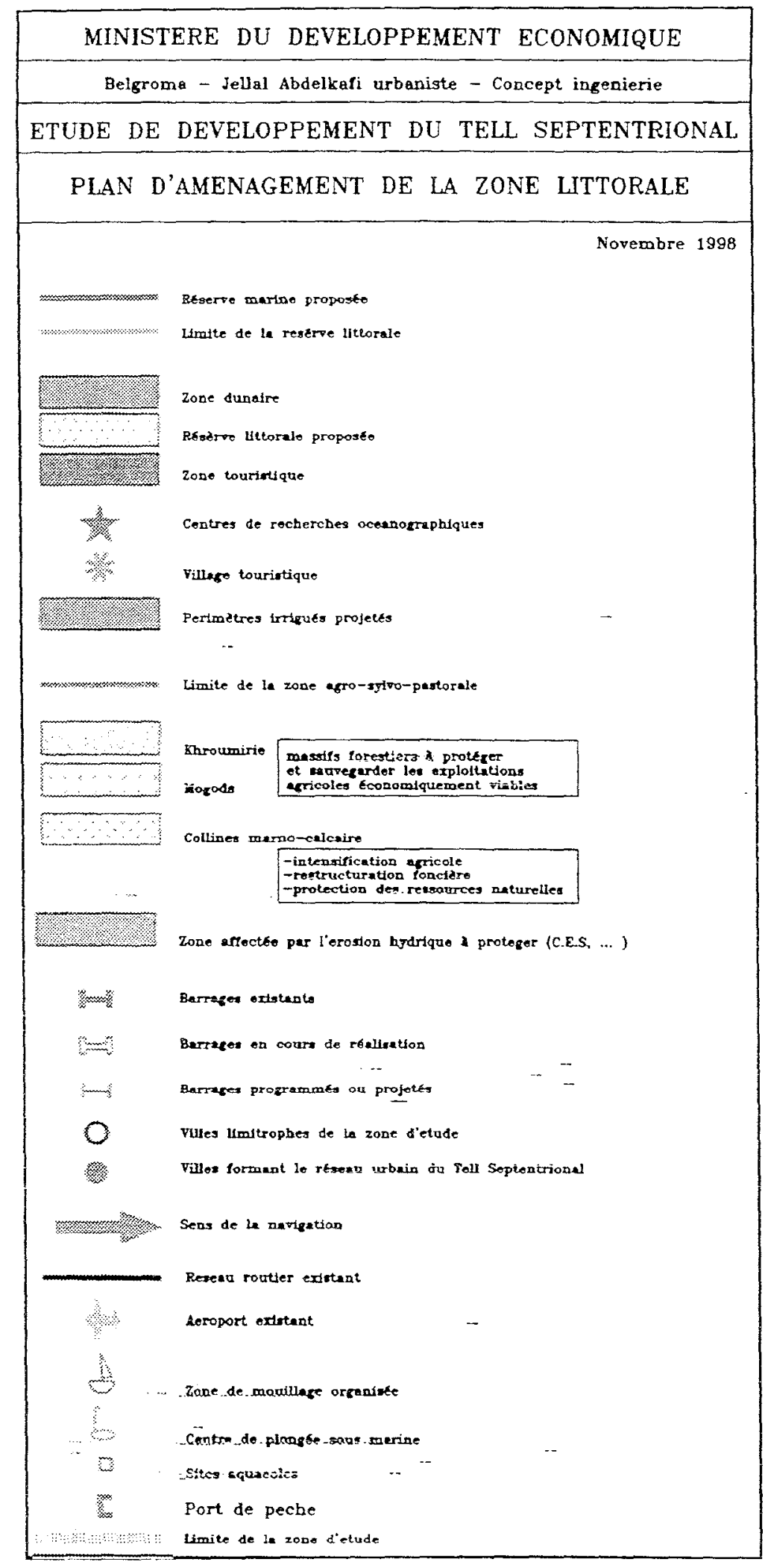

